\title{
Macroscopic Equation and Its Application for Free Ways
}

\author{
Mahmoodreza Keymanesh', Amirhossein Esfahanizad Mousavi \\ ${ }^{1}$ Engineering Faculty, PNU, Tehran, Iran \\ ${ }^{2}$ Transportation of Tehran PNU, Tehran, Iran \\ Email:amirhosseinesfehanizadeh@gmail.com
}

How to cite this paper: Keymanesh, M. and Mousavi, A.E. (2017) Macroscopic Equation and Its Application for Free Ways. Open Journal of Geology, 7, 915-922. https://doi.org/10.4236/ojg.2017.77062

Received: August 13, 2016

Accepted: July 10, 2017

Published: July 13, 2017

Copyright (c) 2017 by authors and Scientific Research Publishing Inc. This work is licensed under the Creative Commons Attribution International License (CC BY 4.0).

http://creativecommons.org/licenses/by/4.0/

\begin{abstract}
Effective transportation systems lead to the efficient movement of goods and people, which significantly contribute to the quality of life in every society. In the heart of every economic and social development, there is always a transportation system. Mathematically the problem of modeling vehicle traffic flow can be solved at two main observation scales: The microscopic and the macroscopic levels. In the microscopic level, every vehicle is considered individually, and therefore, for every vehicle, we have an equation that is usually an ordinary differential equation (ODE). At a macroscopic level, we use from the dynamics models, where we have a system of partial differential equation, which involves variables such as density, speed, and flow rate of traffic stream with respect to time and space. Therefore, considering above content, this study has tried to compare solution of equation of macroscopic flow considering linear form (speed-density) and applying boundary condition that resulting to form solved is non-linear one-order partial differential equation (sharpy method) with non-linear assuming (speed and density) and consequently homographic nonlinear relation (speed-density). The recent case clearly gives more significant speeds than linear case of speed and density that can be a good scientific basis. In terms of safety for accidents and traffic signal, just as a reminder, but it is resulted of the reality that generally solutions of partial differential equations can have different forms. Therefore, the solution of partial differential equation (macroscopic flow) can have different answers and solutions so that all of these solutions apply in PDE (equation of macroscopic flow). Thus, under this condition, we can have solution of linear equation similar to greenberg or greenshield \& android that are explained in logarithm and exponential function, but this article is based mostly on nonlinear solution of macroscopic equation, provided that existing nonlinear relationship between speed and density (homographic the second degree function). As mentioned above, as it gives more reliable and reasonable speeds than greenshield case, it will have more safety. This article has been provided in this field.
\end{abstract}




\section{Keywords}

Freeways, Flow Rates, Density, Average Speed, PDE (Macroscopic Flow)

Nonlinear Resolution of Equation, Homographic Function

\section{Introduction}

As we know, macroscopic models aim at studying traffic flow using a continuum approach, where it is assumed that the movement of individual vehicles exhibits many of the attributes of fluid motion. As a result, vehicle dynamics are treated as fluid dynamics. This idea provides an advantage since detailed interactions are overlooked, and the model's characteristics are shifted toward the more important parameters such as flow rate, concentration, or traffic density, and average speed, all being functions of one-dimensional space and time. This class of models is represented by partial differential equations. Modeling vehicular traffic via macroscopic models is achieved using fluid flow theory in a continuum responding to local or non-local influences [1].

Thus, it is fair to note that partial deferential equation could have numerous answers and have different forms, so that all the answers, in PDE are true, Therefore, with the attention to recent content, it is tired to compare homograph of the second-degree function of nonlinear answer (function of speed \& density) with linear cause (speed and density) which leads to solve PDE by sharpy method of which first state represents more appropriate and more reasonable results that form the basis of this article [2].

\section{Model of Microscopic Traffic Flow (Theory)}

We know there is a close interrelationship between three traffic variables that is density, velocity and traffic flow. Suppose that in the above scenario, cars are moving with constant velocity $\mathrm{v}$, and constant density $D$ such that the distance $d$ between the cars is also constant. Let an observer measure the number of cars $N$ per unit time $t$ that pass (i.e. the traffic flow) [3].

Let $N_{1}$ be the number of cars passing station 1 and $N_{2}$ be the number of cars passing station 2 and $\Delta t$ the duration of the observer counting time. Let $q$ be the flow rate i.e. the number of cars passing a particular station per unit time, then

$$
\begin{gathered}
q_{1}=\frac{N_{1}}{\Delta t}, \quad q_{2}=\frac{N_{2}}{\Delta t} \\
\Delta q=\frac{N_{2}}{\Delta t}-\frac{N_{1}}{\Delta t} \quad \text { or } \quad \Delta N=N_{2}-N_{1}=\Delta q \Delta t
\end{gathered}
$$

For a build-up of cars, therefore, $\Delta N$ will be negative. Thus

$$
\Delta N=(-\Delta q) \Delta t
$$

We assume that $\Delta x$ is short enough so that vehicle density is uniform, then the increase in density during time $\Delta t$ is given by 


$$
\Delta D=-\frac{\Delta N}{\Delta x} \text { or }-\Delta N=\Delta D \Delta x
$$

Since first parties in the relation of (2) and (4) is same so we will have

$$
-\Delta q \Delta t=\Delta D \Delta x \quad \text { or } \quad \frac{\Delta q}{\Delta x}+\frac{\Delta D}{\Delta t}=0
$$

And finally assuming continuously and stretch of Limited extension, equation is obtained as follows

$$
\frac{\partial q}{\partial x}+\frac{\partial D}{\partial t}=0
$$

To solve the above equation, we will assume that answer has been in the form of $V=f(D)$ so that $q=V D$ thus we consider In this case:

$$
\begin{aligned}
& \frac{\partial}{\partial x}(V D)+\frac{\partial D}{\partial t}=0 \\
& \frac{\partial}{\partial x}[D f(D)]+\frac{\partial D}{\partial t}=0
\end{aligned}
$$

Derivative to $x$, we will have:

$$
f(D) \frac{\partial D}{\partial x}+D \frac{\partial f(D)}{\partial x}+\frac{\partial D}{\partial t}=0
$$

Since the $f$ is function $D$ then the derivative $f$ to $D$ would be ordinary thus:

$$
f(D) \frac{\partial D}{\partial x}+D \frac{\mathrm{d} f}{\mathrm{~d} D} \frac{\partial D}{\partial x}+\frac{\partial D}{\partial t}=0
$$

After sorting, we will have:

$$
\left(f(D)+D \frac{\mathrm{d} f}{\mathrm{~d} D}\right) \frac{\partial D}{\partial x}+\frac{\partial D}{\partial t}=0
$$

The above equation is one order nonlinear partial deferential equation (sharpy) $\{1\},\{3\}$.

\section{Calculation}

Now function of speed to density is assumed linear in that case

$$
\begin{aligned}
& V=a+b D \\
& \frac{\mathrm{d} V}{\mathrm{~d} D}=\frac{\mathrm{d} f}{\mathrm{~d} D}=b, \quad f(D)=V
\end{aligned}
$$

Solving the above deferential equation can be written as follows:

$$
\begin{aligned}
& (a+b D+D b) \frac{\partial D}{\partial x}+\frac{\partial D}{\partial t}=0 \\
& (a+2 b D) \frac{\partial D}{\partial x}+\frac{\partial D}{\partial t}=0
\end{aligned}
$$

Now if

$$
D=z, \quad x=x, \quad t=y, \quad \frac{\partial D}{\partial x}=p, \quad \frac{\partial D}{\partial y}=q
$$

The equation can be written in the mathematical form as following 


$$
\begin{gathered}
(a+2 b z) p+q=0 \\
F=(a+2 b z) p+q=0
\end{gathered}
$$

Interpreter Equation of sharpy non-linear equation can be written in general as follows [3]

$$
\frac{\mathrm{d} x}{F_{p}^{\prime}}=\frac{\mathrm{d} y}{F_{q}^{\prime}}=\frac{\mathrm{d} z}{p F_{p}^{\prime}+q F_{q}^{\prime}}=\frac{\mathrm{d} p}{-\left(F_{x}^{\prime}+p F_{z}^{\prime}\right)}=\frac{\mathrm{d} q}{-\left(F_{y}^{\prime}+q F_{z}^{\prime}\right)}
$$

Interpreter equation non-linear partial deferential equation sharpy 12 can be written as follows:

$$
\frac{\mathrm{d} x}{a+2 b z}=\frac{\mathrm{d} y}{1}=\frac{\mathrm{d} z}{(a+2 b z) p+q(1)}=\frac{\mathrm{d} p}{-p \cdot 2 b p}=\frac{\mathrm{d} q}{-q \cdot 2 b p}
$$

With equality of the two last ratios of above equation, we have:

$$
\frac{\mathrm{d} p}{-p \cdot 2 b p}=\frac{\mathrm{d} q}{-q \cdot 2 b p}
$$

And or

$$
\frac{\mathrm{d} p}{p}=\frac{\mathrm{d} q}{q} \Rightarrow q=c p
$$

By substitute the above expression in the differential Equation (12) we have:

$$
\begin{aligned}
& (a+2 b z) p+c p=0 \\
& (a+2 b z+c) p=0
\end{aligned}
$$

Since $p$ cannot be zero, so it is necessary:

$$
p \neq 0: a+2 b z+c=0
$$

And since, according to expression (10) $D=Z$ were supposed to conclude from the relationship

$$
2 b D-a=-c
$$

And, finally, speed function with density with respect to the relations (8) and (17) can be written according to the following equation:

$$
V=-c-b D
$$

Recent relation shows linearity assumption (speed \& density) that coefficients $b, c$ determined based of quality of traffic.

For the second case, it is assumed the relationship speed and density function of homographic of the second degree function, have the following meaning:

$$
f(D)=V=\frac{a D^{2}+b D+c}{D}, \quad \frac{\mathrm{d} V}{\mathrm{~d} D}=\frac{\mathrm{d} f}{\mathrm{~d} D}=\frac{a D^{2}-c}{D^{2}}
$$

With placement relations (19). In (7) we have:

$$
(2 a D+b) \frac{\partial D}{\partial x}+\frac{\partial D}{\partial t}=0
$$

Now according to expression (10), the above equation can be written as follows:

$$
(2 a z+b) p+q=0
$$


And or

$$
F=(2 a z+b) p+q=0
$$

As the first argument to resolve the problem non-linear one order partial differential equation (Charpy) we will have $q=c p$.

And by replacement recent value in (21) concluded:

$$
b=-2 a D-c
$$

Finally, by letting above relation in Equation (21) speed function obtains in following form.

$$
V=\frac{-a D^{2}-c D+c}{D^{2}}
$$

Above function indicates relation (speed-density) with assuming non-linearity, that the coefficients $a, c$, depending on kind of traffic quality. So that this parameters will determinate in here.

Acts of Boundary conditions:

Now determining the coefficients $b, c$ based on relations (18). For each line on Freeway in terms Quality of traffic and the following conditions, we can have $\{4\}$

$$
\left\{\begin{array} { l } 
{ D = 7 } \\
{ V = 1 2 0 }
\end{array} \quad \left\{\begin{array}{l}
D=28 \\
V=85
\end{array}\right.\right.
$$

Coefficients $b, c$, respectively, will be equal with:

$$
b=1.67, \quad c=131.7
$$

Therefore, the relationship of speed function \& density can be written as follows:

$$
V=131.7 D-1.67 D^{2}
$$

And:

$$
\begin{aligned}
& q=V \cdot D \\
& q=131.7 D-1.67 D^{2}
\end{aligned}
$$

Maximum amount of the flow rate for each line of freeway, basses (26) is determined as follows.

$$
\begin{aligned}
& \frac{\mathrm{d} q}{\mathrm{~d} D}=0, \quad D=\frac{131.7}{2 \times 1.67}=39.43 \\
& q_{\max }=131.7 \times 39.43-1.67 \times(39.43)^{2}=2597
\end{aligned}
$$

Now To determine the coefficients $a, c$ based on relation (24) for each line of freeways in terms quality of traffic in following conditions can be written: $\{4\}$

$$
\left\{\begin{array} { l } 
{ D = 7 } \\
{ V = 1 2 0 }
\end{array} \quad \left\{\begin{array}{l}
D=28 \\
V=85
\end{array}\right.\right.
$$

The coefficients $a, c$, respectively, will be equal with:

$$
a=2.485, \quad c=-160.3
$$

Thus, function of velocity-density is written as follows: 


$$
V=\frac{-2.485 D^{2}+160.3 D-160.3}{D}
$$

For two answers based on relations (27) and (25) we can form following Table 1.

And for second cause, it was assumed non-linear relationship between speed $\&$ density and was indicated for homographic of the second degree function, thus we can have following Table 2 .

By comparing above tables it is clearly by linearity assumption of velocity and density respectively. For two consecutive service level we face with reduction of velocity almost 1o kilometer per hours. But based on non-linearity of velocity and density, speed reduction for two consecutive service level will be non-uniform now. The question that arises is: Which one of the macroscopic traffic flow patterns is more appropriate? With the attention to above points we can have the following results. But initially, we investigate the other velocity and density models as follows [4].

\section{The Other Models of Density, Velocity}

The Greenshields Model (Greenshields, 1935) is a simple and widely used model. It is assumed that the velocity is a linearly decreasing function of the traffic flow density, and it is given by: [5]

$$
V=V_{f}\left(1-\frac{D}{D_{\text {jam }}}\right)
$$

In which the $V_{f}$ is free speed. $D=$ density $D_{\text {jam }}$ is the jam density.

According to above expression when speed is equal with zero density is equal

Table 1. Density, capacity, speed.

\begin{tabular}{cccc}
\hline LOS & Density $(d)$ & Capacity $(q)$ & Speed $(v)$ \\
\hline$A$ & 7 & 840 & 120 \\
$B$ & 11 & 1243 & 113 \\
$C$ & 16 & 1680 & 105 \\
$D$ & 22 & 2090 & 95 \\
$E$ & 28 & 2380 & 85 \\
Heavy traffic & 40 & 2600 & 65 \\
\hline
\end{tabular}

Table 2. Density, capacity, speed.

\begin{tabular}{cccc}
\hline LOS & Density $(D)$ & Capacity $(q)$ & Speed $(v)$ \\
\hline$A$ & 7 & 840 & 120 \\
$B$ & 11 & 1298 & 118 \\
$C$ & 16 & 1760 & 110 \\
$D$ & 22 & 2156 & 98 \\
$E$ & 28 & 2380 & 85 \\
Heavy traffic & 40 & 2280 & 57
\end{tabular}


with saturated density, Conversely when density is zero, the speed will be free velocity.

Greenberg model: Greenberg developed a model of speed-density showing a logarithmic relationship. In Greenberg's model the speed-density function is given by:

$$
V=V_{f}-L_{n}\left(\frac{D}{D_{\mathrm{jam}}}\right)
$$

Underwood Model: Underwood model where the velocity-density function is represented as follows:

$$
V=V_{f} \times \mathrm{e}^{-\frac{D}{D_{\mathrm{jam}}}}
$$

Eddy Model: This model basically combines Greenberg's and Underwood's model. Eddie suggested that for densities $D<50$ as follows:

$$
V=54.9 \mathrm{e}^{\frac{D}{163.9}}
$$

And finally, for $D>50$ speed function will be determined as follows:

$$
V=28.6 \ln \left(\frac{162.6}{D}\right)
$$

\section{Conclusion}

According to Table 1 and Table 2 based on relations (27) and (25), it can be inferred in the linearity assumption (speed \& density) and with the attention to speed depending on the density, speed monotonically decreases with density. This is far from reality and is not sufficiently accurate, but in the non-linear case (speed \& density), speed does not comply uniform increasing with density and this is compatible with this fact. As for low densities, the amount of velocity is closer to free speed, which is reasonable. But in linearity assumption for all densities, speed uniformly decreases. This is far from reality and is not sufficiently accurate. To prove the claim for high densities which will lead to traffic jams, specifically, for example, $D=40$ and linearity assumption (speed \& density), value of speed $V=65 \mathrm{~km} / \mathrm{hr}$ are obtained therefore, we have $q=V D=2600$ for value of capacity, the same conditions for non-linearity assumption (speed \& density), and $D=40$, we have $V=57 \mathrm{~km} / \mathrm{hr}$ and finally for capacity, we obtain $q=V D=2300$. Thus, it is inferred from above paragraphs in linearity assumption for high densities, we obtained high velocity, thus with the attention to volume, is proportional to the product speed and density. Therefore, volume increases also with speed increases. This subject created false capacity for line, which is far from reality. But nonlinearity assumption and high densities capacity don't highly accelerate. And almost the amount of capacity was limited of about 2300 vehicles per hour, which is more logical. As low densities in non-linearity assumption speed are closer to free speed, this is reasonable. Thus actual capacity is caused. But for linearity assumption, speed reduces with density uniformly, it seems unreasonable. As for non-linearity assumption, variation 
of speed was complied in non-uniform. That is true. Therefore, according to authors and with attention to partial differential equation, it could have general-

ly different and various answers, thus non-linear cause of (speed and density) compared to the linearity assumption seems to be practical and logical application. This paper has been prepared and set in this regard.

\section{References}

[1] Papageorgiou, G. and Maimaris, A. (2010) Modelling Simulation Methods for Intelligent Transportation Systems. European University Cyprus, 20, 53-65.

[2] Ramos, A., Ferreiera, J. and Barcelo, J. (2012) Modeling \& Simulation for Intelligent Transportation Systems, Skoog.

[3] Wesley, J. (2004) Social Participation and Disciplinary Care for Everyone. Translated by Dr. Masoudnia, A., Second and Third Studies, Social Security Number, 183.

[4] Chakrabarty, B.K. (2001) Urban Management, Concepts, Principles, Techniques and Education. Cities, 18, 331-345.

[5] Harvey, D. (2009) Social Justice and the City. 2nd Edition, the University of Georgia Press, Athens.

\section{Submit or recommend next manuscript to SCIRP and we will provide best} service for you:

Accepting pre-submission inquiries through Email, Facebook, LinkedIn, Twitter, etc. A wide selection of journals (inclusive of 9 subjects, more than 200 journals)

Providing 24-hour high-quality service

User-friendly online submission system

Fair and swift peer-review system

Efficient typesetting and proofreading procedure

Display of the result of downloads and visits, as well as the number of cited articles

Maximum dissemination of your research work

Submit your manuscript at: http://papersubmission.scirp.org/

Or contact ojg@scirp.org 